\title{
Expectations of consistency about the self: Consequences for self-concept formation
}

\author{
Allen R. McConnell, ${ }^{\mathrm{a}, *}$ Robert J. Rydell, ${ }^{\mathrm{a}}$ and Jill M. Leibold ${ }^{\mathrm{b}}$ \\ a Department of Psychology, Miami University, 110D Benton Hall Oxford, OH 45056, USA \\ ${ }^{\mathrm{b}}$ Michigan State University, USA
}

Received 14 June 2001; revised 28 December 2001

\begin{abstract}
This study proposes that the on-line versus memory-based judgment distinction has important implications for understanding self-concept formation and content. Past research has shown that perceivers form on-line judgments of targets who are expected to display a great deal of behavioral consistency (e.g., individuals) but form memory-based judgments of targets who are expected to display less behavioral consistency (e.g., groups). Experiment 1 found that perceptions of behavioral consistency for the self were strong and closer to perceptions of consistency for individuals than for groups. Thus, we predicted that self-concepts should, by default, be formed on-line. Experiment 2 supported this prediction. Experiment 3 manipulated participants' expectations of behavior consistency and found that those expecting greater consistency in their behaviors formed on-line self-concepts. Experiment 4 used a different methodology and behavioral domain, and it collected more direct process data that further supported the importance of the on-line versus memory-based distinction for the self. Implications for understanding self-concepts and for comparing self-knowledge to how people understand other types of social entities are discussed.
\end{abstract}

(C) 2002 Elsevier Science (USA). All rights reserved.

Know thyself

Temple of Apollo at Delphi, 6th century BC

This Delphic maxim has been recited by philosophers and parents alike over the ages. At the heart of this adage is that people should know their attributes, abilities, and attitudes that are self-defining. In other words, they should know their self-concept. But how do people form self-concepts? The current work examines some of the basic cognitive processes involved in self-concept formation.

Clearly, there are many sources of information for self-concept formation, including self-perception (e.g., Bem, 1972), social comparison (e.g., Wills, 1981), cultural influences (e.g., Markus \& Kitayama, 1991), and feedback from others both real (e.g., Cooley, 1902) and anticipated (e.g., Mead, 1934). Once formed, self-concepts can have important implications for one's self-esteem (e.g., Pelham, 1995), behavior (e.g., Bandura,

\footnotetext{
${ }^{*}$ Corresponding author.

E-mail address: mcconnar@muohio.edu (A.R. McConnell).
}

1986), outlook on life (e.g., Taylor \& Brown, 1988), mental regulation (Renaud \& McConnell, 2002), and experience of emotion (e.g., Higgins, 1997). Thus, a great deal is known about the sources that contribute to self-concept formation and the important implications of the already-formed self-concept.

In addition to these efforts, other lines of research have examined the cognitive representation of self-relevant knowledge. For example, Rogers, Kuiper, and Kirker (1977) found that recall of information associated with the self is much better than when the same information is not associated with the self. Subsequent work, however, suggests that this self-reference effect is mostly due to the greater organization, elaboration, and amount of information associated with the self (Bower \& Gilligan, 1979; Klein \& Loftus, 1990) instead of unique properties of the self (for reviews, see Greenwald \& Banaji, 1989; Kihlstrom \& Klein, 1994). In addition to exploring the ability of the self to serve as a retrieval vehicle, research has examined whether recall of selfknowledge is based on specific instances (i.e., exemplars) or is based on traits (i.e., prototypes) in memory. Work 
by Klein and colleagues (e.g., Klein \& Loftus, 1993; Klein, Loftus, Trafton, \& Fuhrman, 1992; Klein, Sherman, \& Loftus, 1996) using a task-facilitation paradigm supported a mixed-model where specific instances are retrieved for domains that are relatively low in self-descriptiveness, but where abstracted traits are retrieved for domains that are relatively high in self-descriptiveness. This research suggests that the content of what is recalled about the self may vary across time and situations.

Although the above research speaks to the richness of the self as a memory aid and to the content of recall about the self, little is known about the processes by which self-relevant information is initially utilized. In other words, how is self-relevant information processed as it is received? How do expectations about the self and the availability of cognitive resources result in different information processing outcomes for self-relevant information? What are the implications of these processes? How might these processes relate to our knowledge about how people come to understand other social entities, such as individuals and groups?

To explore these questions, we suggest that it is useful to consider how people form impressions about other social entities. Several programs of research have explored how people form impressions about individuals (McConnell, 2001; McConnell, Sherman, \& Hamilton, 1994b, 1997b; Srull, 1981; Srull, Lichtenstein, \& Rothbart, 1985) and members of groups (Hamilton \& Gifford, 1976; McConnell et al., 1994b, 1997b; Srull et al., 1985). This body of work has shown that people demonstrate some systematic tendencies when integrating information about particular types of social targets. However, there are conditions under which perceivers adopt different information processing goals, which in turn influence how perceptions are formed for similar targets. This work suggests that it is useful to think about a continuum of social information processing possibilities ranging from highly integrative information processing (where on-line impressions are formed) to less integrative information processing (where memorybased impressions are rendered).

In this context, on-line judgments occur when a perceiver forms and actively organizes an integrative impression while relevant information is being gathered, whereas memory-based judgments occur when a perceiver does not form an impression until the time that a judgment is required (Hamilton \& Sherman, 1996; Hastie \& Park, 1986). For example, McConnell et al. (1994b) found that perceivers, typically, form on-line judgments of individual social targets, but render memory-based judgments about group targets. It was hypothesized that people adopt different goals for understanding social targets, and thus were more likely to form on-line impressions of individuals, relative to groups, because they expect greater consistency in the behaviors of a single individual than in the behaviors of members of a group. This reasoning is consistent with findings in the person memory literature as well (e.g., Srull, 1981; Srull et al., 1985; for reviews, Stangor \& McMillian, 1992; Srull \& Wyer, 1989). Without an expectation of behavioral consistency, forming a coherent on-line impression would seem relatively useless.

The hypothesis that expectations of target consistency affect how social information is processed was more directly tested by McConnell et al. (1997b), who directly manipulated participants' expectations of behavioral consistency for individual and group social targets by instruction set. When participants were not provided with expectations of consistency, strong differences emerged between individuals and groups, reflecting the natural default differences between these social targets. However, when expectancies of consistency were explicitly given to participants, the type of target encountered was less important and participants revealed on-line judgments for high consistency targets (both individual and group targets) and memory-based judgments for low consistency targets (both group and individual targets). These studies suggest that different types of social targets may reveal default differences in on-line and memory-based judgments. However, the critical determinant of social information processing mechanism hinges on expectations of target consistency (McConnell et al., 1997b; see also, McConnell, 2001), which in turn evoke different processing goals for forming impressions about social targets (McConnell et al., 1994b).

\section{Expectations of consistency for the self}

Most research in social perception has focused on groups and individuals as social targets (e.g., Lichtenstein \& Srull, 1987; McConnell et al., 1994b, 1997b; Srull, 1981; Srull et al., 1985), however, very little is known about how self-relevant information is processed. Although individuals often hold overly positive beliefs about themselves (Taylor \& Brown, 1988) and prefer to strike a balance between being similar to others while being somewhat unique (Markus \& Kunda, 1986), little is known about individuals' perceptions of self consistency. Because expectations about a target's behavioral consistency shape social information processing about it, an important question to address is how much consistency do people expect from themselves.

Baxter and Goldberg (1987) attempted to explore this question by asking students to rate themselves and to rate a close friend in terms of how well 60 personality traits described themselves and their friend. They found that participants rated themselves as more variable than their close friend on $72 \%$ of the traits. Their study suggests that people may expect less consistency in their own behavior than in the behavior of another. However, 
such a conclusion may not be unequivocal. For instance, the choice of a same-sex person who the participants knew very well and liked may not represent the best choice of a comparison person. Also, the authors did not assess consistency for the self in comparison to perceptions of consistency for a social target (e.g., groups) that induce memory-based judgments (McConnell et al., 1994b, 1997b). Therefore, it is unclear whether the observed differences between oneself and a close, samesex, well-liked friend generalize to differences between oneself and a nondescript target individual. Further, it is unclear whether these differences are small or sizable in magnitude when compared to targets that evoke different social information processing outcomes than individual targets.

On theoretical grounds, there are reasons to predict that people may expect relatively little consistency in their own behaviors. For example, the classic actorobserver effect suggests that people expect that their own behavior is determined by multiple external sources whereas the locus of others' behaviors is internal in nature (Jones \& Nisbett, 1972). Moreover, because individuals have greater access to their own behavior across a variety of contexts relative to other social entities (e.g., Heider, 1958), they may be aware of greater fluctuation in their own actions and thus perceive greater variability for the self than for others.

Conversely, there are sensible reasons to predict that people expect a great deal of consistency in their own behaviors. Because people often need to predict, control, and develop their skills and abilities (e.g., Swann, Stein-Seroussi, \& Giesler, 1992; Trope \& Efrat, 1994), they need to have a relatively clear sense of who they are. Although self-assessment goals do not necessitate that people believe that they exhibit consistency, it does require that they not have an overly diffuse sense of their own qualities. Another reason why people might expect consistency in their own behavior is that in order for self-enhancement biases to be effective (e.g., Weinstein, 1980), one must perceive a relatively stable, positive self. Put simply, it would be difficult for one to maintain positive self-illusions while believing that the self is tremendously variable. Also, although people may exhibit variability in their behavior, they show considerable behavioral stability in domains with common psychological features (Shoda, Mischel, \& Wright, 1994) and are aware of the extent to which they show this intra-individual stability (Mischel \& Shoda, 1995). Finally, construals of the self in Western cultures should lead individuals to believe that the self is relatively stable, constrained, and unitary (Fiske, Kitayama, Markus, \& Nisbett, 1998; Markus \& Kitayama, 1991). Therefore, there are several reasons to predict that people should expect a relatively strong degree of consistency for the self, including ones that serve many self motives.
Because one could reasonably argue that people should expect either minimal or sizable consistency for themselves relative to other social targets, Experiment 1 assessed the extent to which people expect consistency in their own behaviors relative to targets that have been shown by previous research to evoke expectations of sizable consistency (nondescript individuals) and to evoke expectations of minimal consistency (nondescript groups). If people expect sizable consistency for the self (like they do for nondescript individuals), we would predict that self-concepts will, typically, be formed online. On the other hand, if people expect minimal consistency for the self (like they do for nondescript groups), we would expect that self-concepts will, typically, be memory-based. Experiments 2-4 examined the implications of these predictions in greater detail.

\section{Experiment 1: Expectations of consistency for the self, individuals, and groups}

We were interested in examining the extent to which people expect consistency in their own behaviors relative to social targets that have been shown to elicit strong expectations of consistency and on-line impressions (individuals) and to elicit weak expectations of consistency and memory-based judgments (groups). Participants were asked to consider a series of traits and to assign the number of days in an average year that a social target (the self, an individual, or members of a social group) exhibits that trait across seven different levels of extremity. They recorded their judgments using the computer interface displayed in Fig. 1, and means and standard deviations were computed based on their distributions. Although it was expected that participants would reveal self-enhancing biases (e.g., report that their mean level of intelligence is greater than the mean level of intelligence for a nondescript individual), we were primarily interested in the variability revealed in their distributions. Specifically, we examined whether the variability for the self would be closer to the variability produced for the individual social target or closer to the variability produced for the group social target. Further, we expected that participants would report less consistency for the group social target than for the individual social target, replicating previous work showing that perceivers expect greater behavioral consistency from individuals than they do from groups (McConnell et al., 1997b).

\section{Method}

\section{Participants}

A sample of 66 undergraduates participated in return for research credit in their introductory psychology courses. 


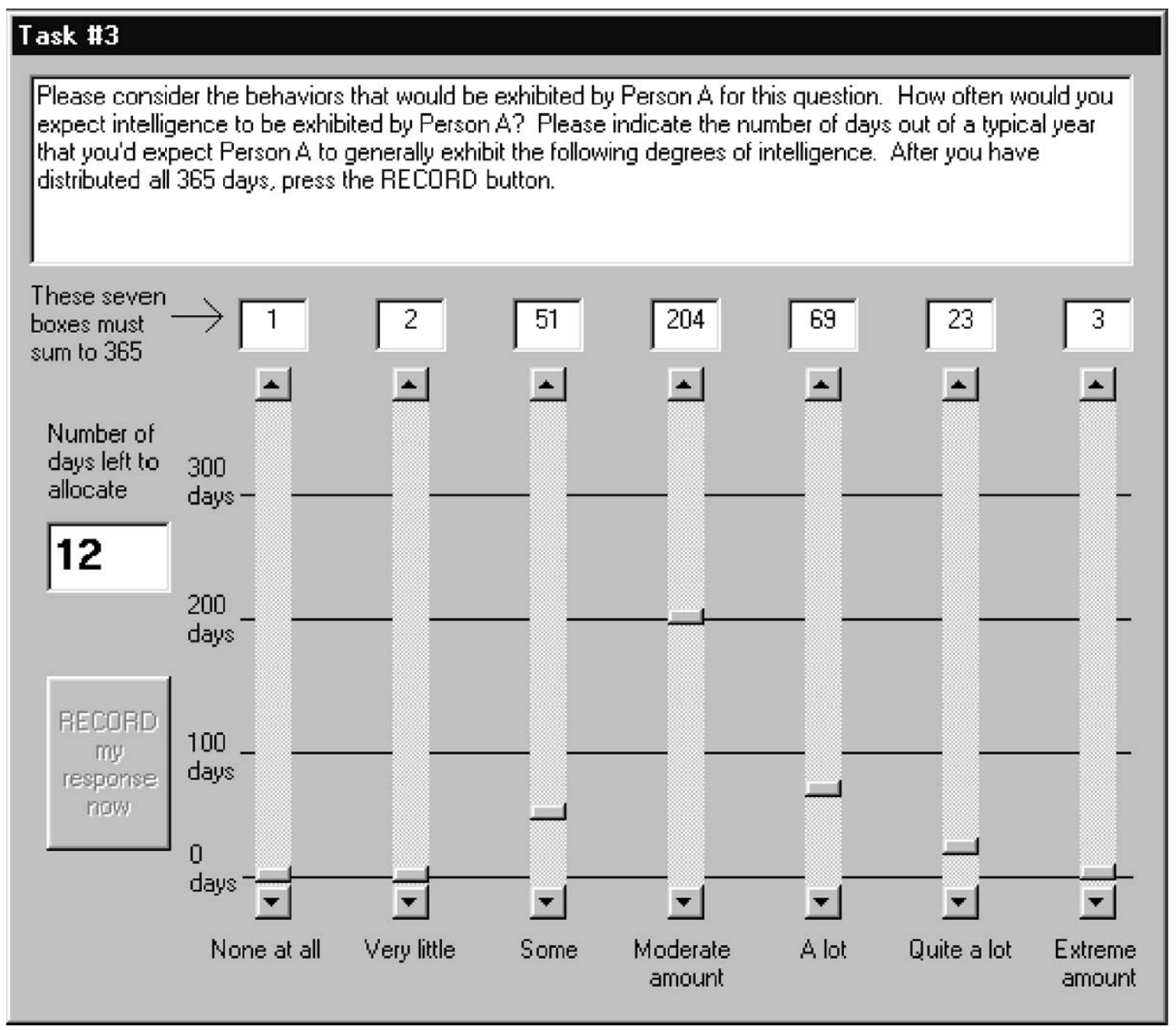

Fig. 1. Interface used for recording participants' distribution of behaviors across the seven extremity levels in Experiment 1.

\section{Procedure}

Participants completed the experiment at computer workstations in individual rooms. They considered each of five traits (intelligence, selfishness, insightfulness, creativity, and considerateness) separately for each of three social targets (themselves, a nondescript person, and a nondescript group). The participants responded to all five traits (order of trait presentation was randomly determined for each participant) for one social target before moving to the next social target. The order of social target presentation was randomly determined (self-person-group, self-group-person, person-groupself, and so forth). Analyses revealed that this order variable produced no main or interactive effects on any of the measures, and thus it receives no further discussion.

Participants were asked to consider the range of behaviors performed by each social target and to report the distribution of that trait exhibited by the target across the course of a year using the interface displayed in Fig. 1. They distributed 365 days worth of behaviors across seven levels (ranging from "not at all" to "extreme amount") for each judgment. Thus, each participant recorded 15 distributions ( 5 traits $\times 3$ targets).

To manipulate the target type, participants were told to (self target condition) "consider yourself," (person target condition) "consider a person chosen at random from the general population-we'll refer to this target as Person A," or (group target condition) "consider various members of a group chosen at random from the general population-we'll refer to this target as Group A." Thus, the person target and group target were nondescript in nature, making comparisons possible to previous research examining default social information processing differences between individual targets and group targets.

For each judgment, participants adjusted the sliders on the computer interface to allocate the 365 days across the seven levels of behaviors, which were converted to a scale ranging from 1 (not at all) to 7 (extreme amount) for each judgment. The number of days assigned to each level represented the number of observations at that 
level. For each of the participants' 15 distributions, a mean and a standard deviation score were computed.

\section{Results}

\section{Analyses of means (self-serving biases)}

To assess whether participants showed evidence of self-serving biases, the means for each of the five traits were analyzed in a one-way (target type: self, person, or group) within-subjects analysis of variance (ANOVA). Table 1 displays the results. Self-serving biases were exhibited for four of the five traits (i.e., intelligence, selfishness, insightfulness, and considerateness). Although the means for creativity were in the predicted direction, the main effect did not achieve significance. Post-hoc analyses revealed that participants rated themselves as more intelligent, less selfish, more insightful, and more considerate than Person A. There were no differences between mean ratings of Person A and Group A except for selfishness, where the members of Group A were seen as more selfish than Person A, who in turn was seen as more selfish than the self. Thus, as expected, participants showed strong evidence of selfserving biases.

\section{Analyses of standard deviations (expectations of target consistency)}

To assess whether participants expected their own behavior to be relatively consistent (and thus low in variability, like individuals) or relatively inconsistent (and thus high in variability, like groups), the standard deviations for each of the five traits were analyzed in a one-way (target type) within-subjects ANOVA. The results are presented in Table 1, revealing main effects for all five traits. Post-hoc analyses indicated that expectations of consistency for the self were always greater (i.e., lower variability) than expectations of group consistency. That is, expectations of consistency for the self more closely mirrored those of Person A than of Group A. In cases where there were differences between ratings of self consistency and Person A consistency (i.e., intelligence, selfishness, and considerateness), expectations of consistency for the self were always greater than expectations of consistency for Person A. Thus, these data strongly support the prediction that people expect relatively strong consistency in their own behaviors. Also, these data provide further evidence that people expect greater consistency in the behavior of an individual than they do in the behavior of members of groups.

\section{Discussion}

Experiment 1 demonstrated that people do expect strong consistency in their own behaviors relative to other social targets, especially groups. Participants also revealed strong self-serving biases as well. Because previous work showing that impressions of targets expected to reveal behavioral consistency are formed on-line (McConnell et al., 1994b, 1997b), we predicted that selfconcepts would typically be formed on-line as well. Experiment 2 tested this prediction, and Experiment 3 manipulated expectations of consistency for the self to examine a causal relation for these outcomes. Lastly, Experiment 4 examined the role of cognitive resources and collected additional process measures to speak more directly to the hypothesized underlying mechanism.

Table 1

Average mean and standard deviations for traits as a function of target type in Experiment 1

\begin{tabular}{|c|c|c|c|c|}
\hline & \multicolumn{3}{|c|}{ Target type } & \multirow[b]{2}{*}{$F(2,130)$} \\
\hline & Self & Person & Group & \\
\hline \multicolumn{5}{|l|}{ Intelligence } \\
\hline Standard deviation & $1.06_{\mathrm{a}}$ & $1.17_{\mathrm{b}}$ & $1.28_{\mathrm{c}}$ & $10.57^{* *}$ \\
\hline \multicolumn{5}{|l|}{ Selfishness } \\
\hline Mean & $2.81_{\mathrm{a}}$ & $3.56_{\mathrm{b}}$ & $3.88_{\mathrm{c}}$ & $27.41^{* *}$ \\
\hline Mean & $4.28_{\mathrm{a}}$ & $3.80_{\mathrm{b}}$ & $3.96_{\mathrm{b}}$ & $8.39^{* *}$ \\
\hline Standard deviation & $1.16_{\mathrm{a}}$ & $1.22_{\mathrm{a}}$ & $1.32_{\mathrm{b}}$ & $5.83^{* *}$ \\
\hline \multicolumn{5}{|l|}{ Creativity } \\
\hline Mean & $4.31_{\mathrm{a}}$ & $4.01_{\mathrm{a}}$ & $4.13_{\mathrm{a}}$ & 2.22 \\
\hline Standard deviation & $1.20_{\mathrm{a}}$ & $1.27 \mathrm{a}$ & $1.34_{b}$ & $4.56^{*}$ \\
\hline \multicolumn{5}{|l|}{ Considerateness } \\
\hline
\end{tabular}

Note. Means in a row that do not share a common subscript differ at the .05 level based on Fisher's protected least significant difference (PLSD) test.

${ }_{* *}^{*} p<.05$.

${ }^{* *} p<.01$. 
Experiments 2-4 employed experimental paradigms that allowed us to determine whether participants formed on-line, or memory-based, self-concepts. When people form on-line impressions, their evaluations are formed while they initially encounter and encode target-relevant behaviors. During this process, early information is especially influential in target evaluations, producing primacy effects in judgment (Dreben, Fiske, \& Hastie, 1979). In contrast, memory-based judgments are not rendered until the time that judgment is required (Hastie \& Park, 1986; Sherman, Zehner, Johnson, \& Hirt, 1983). Rather than forming an on-going impression, perceivers encode but do not integrate the behavioral information. As a result, memory-based judgments about social targets are more influenced by recent events because recently encountered behaviors will be relatively more available in memory when integrative processing does not occur. Thus, one can infer whether on-line or memory-based self-concepts are formed based on whether self-relevant evaluations reveal primacy or recency effects. Experiment 4 examined primacy and recency effects in judgments about the self using a different paradigm in a different behavioral domain, and it also collected additional, independent memory measures to speak more directly to the hypothesized underlying processes.

\section{Experiment 2: Default self-concept formation processes}

To examine the presence of primacy and recency effects, participants in Experiments 2 and 3 performed an analogy task and were provided with noncontingent feedback about their performance. Pretesting established that participants were relatively uncertain about their skill at performing analogies, allowing us to convincingly manipulate feedback about their performance to observe its effects on their perceptions. After completing each analogy item, they were given feedback indicating that they either did, or did not, solve the item correctly. In all cases, participants' performance feedback was identical (they were told that they correctly solved 16 of 24 analogies). Adopting a methodology similar to one used by McConnell, Sherman, and Hamilton (1994a), we manipulated the sequential presentation of feedback while keeping overall performance feedback constant. As Table 2 shows, between-subject conditions either placed most of the correct responses early in the task (a primacy condition), placed most of the correct responses late in the task (a recency condition), or spread the correct responses evenly throughout the task (a balanced condition). After completing all 24 items, participants were asked to provide estimates of how many items they correctly solved (a performance measure) and were asked to provide ratings of their skill at solving analogies in general (an ability measure). The former measure allowed us to assess their perceptions of
Table 2

Distribution of correct and incorrect responses as a function of presentation condition in Experiments 2-3

\begin{tabular}{lll}
\hline & \multicolumn{2}{l}{ Sequential presentation by halves } \\
\cline { 2 - 3 } Conditions & First half (items 1-12) & Second half (items 13-24) \\
\hline Primacy & 10 Correct, 2 Incorrect & 6 Correct, 6 Incorrect \\
Balanced & 8 Correct, 4 Incorrect & 8 Correct, 4 Incorrect \\
Recency & 6 Correct, 6 Incorrect & 10 Correct, 2 Incorrect \\
\hline
\end{tabular}

the events that occurred during the analogies task, and the latter measure allowed us to assess whether their task experiences generalized to more global judgments about their ability.

Evidence of on-line self-concept formation would be revealed by participants reporting the best performance and greatest ability in the primacy condition, reflecting primacy effects in judgments. Accordingly, evidence of memory-based self-concept formation would be indicated by participants reporting the best performance and greatest ability in the recency condition, demonstrating recency effects in judgments. Thus, betweencondition differences in perceptions of performance and ability would reflect the nature of self-concept formation, on-line versus memory-based. In addition to providing evidence for the process employed in their judgments, these outcomes demonstrate an important implication of forming on-line and memory-based selfconcepts. That is, between-subject condition differences would show how differential perceptions of objectively equivalent feedback result from differences in processing self-relevant information.

Experiment 1 revealed that participants expect a great deal of consistency in their own behaviors, thus we predicted that participants in Experiment 2 would form on-line self-concepts. This outcome would be revealed by participants estimating the greatest performance and ability in the primacy condition (where early feedback was especially positive) and the lowest performance and ability in the recency condition (where early feedback was especially negative).

\section{Method}

\section{Participants and design}

A sample of 81 undergraduates participated in return for extra credit in their courses. They were randomly assigned to either a primacy, balanced, or recency feedback condition (27 participants per condition). None of them reported any suspicion about the study or the validity of the feedback provided to them on the analogy items.

\section{Stimulus materials}

Our goal was to develop analogy items that were moderately difficult in order to maximize participants' 
uncertainty about their performance, allowing us to provide noncontingent feedback. A large pool of moderately difficult multiple-choice analogy problems was developed. For each analogy problem, a target pair of words was presented, followed by five pairs of words numbered from 1 to 5 , where one choice was an objectively correct answer. Participants were asked to identify the pair of words from the five choices that most closely represented the relationship to the target pair.

During pretesting, undergraduates (none of whom participated in the current study) attempted to solve each analogy item. After providing their response to an analogy item, they estimated their confidence about the accuracy of their answer. From this pretesting pool, 24 analogy items were selected where participants' confidence levels were relatively low and were uncorrelated to their actual performance. This lack of correlation between actual accuracy and subjective confidence ensured that manipulated performance feedback could be given about the analogy items. That is, participants' subjective sense of performance would be unrelated to their actual performance, making noncontingent feedback credible.

\section{Procedure}

Upon arrival, participants completed consent forms, were randomly assigned to conditions, and completed the remainder of the experiment alone at computer workstations in individual rooms. The computer informed participants that the purpose of the study was to learn how people solve analogy problems. An example of an analogy problem was provided along with an explanation of the nature of analogy problems. Participants then completed the 24 analogy problems, which were presented in a randomly determined order. After they responded to each analogy item, noncontingent feedback was given, indicating that their answer was "correct" or "incorrect."

Although all participants were provided with the same overall feedback (16 items correct and 8 items incorrect), the sequential presentation of this feedback was varied as part of the between-subjects manipulation illustrated in Table 2. After completing all of the analogy items, participants were told that they had completed 24 analogy items, and they estimated the number that they solved correctly. This estimate reflected their perception of their performance on the analogy task. Next, participants were asked to estimate their general skill in solving analogies on a 1 (very unskilled) to 9 (very skilled) scale, which assessed their perception of general ability in solving analogies.

\section{Results}

\section{Performance estimate}

A one-way ANOVA was performed on the participants' estimate of the number of analogies they correctly
Table 3

Performance and skill means as a function of presentation condition in Experiment 2

\begin{tabular}{lccc}
\hline & \multicolumn{3}{c}{ Presentation condition } \\
\cline { 2 - 4 } Measure & Primacy & Balanced & Recency \\
\hline Perceived performance & $17.48_{\mathrm{a}}$ & $16.41_{\mathrm{a}, \mathrm{b}}$ & $15.23_{\mathrm{b}}$ \\
Perceived skill & $6.29 \mathrm{a}$ & $5.56_{\mathrm{b}}$ & $5.56_{\mathrm{b}}$ \\
\hline
\end{tabular}

Note. Means in a row that do not share a common subscript differ at the .05 level based on Fisher's PLSD tests.

solved using presentation condition as the independent variable. Because on-line self-concept formation was expected, it was predicted that performance estimates would be larger in the primacy condition than in the recency condition. As Table 3 reports, the predicted effect of presentation condition was found, $F(2,78)=$ $7.30, p<.01$, revealing that participants reported greater estimates of performance in the primacy condition than in the recency condition. These results indicate that people relied strongly on initial feedback and formed on-line self-concepts.

\section{Ability estimate}

To examine whether the feedback pattern affected general beliefs about analogy skill and not just beliefs about performance on this particular task, a one-way ANOVA was conducted on the ability estimates. As with the performance estimates, it was expected that the formation of on-line self-concepts would be revealed by greater estimates of ability in the primacy condition than in the recency condition. Consistent with predictions, a marginal main effect of presentation condition was revealed, $F(2,78)=2.85, p<.07$. Table 3 reports that participants tended to report greater estimates of their ability in the primacy condition than in the other two conditions.

\section{Objective performance}

To ensure that participants' between-condition differences in perceptions of performance and ability were not based on actual differences in their performance, the number of analogy items that participants actually solved correctly was analyzed in a one-way ANOVA using presentation condition as the independent variable. As expected, no effect of presentation condition was found, $F(2,78)=.05$, ns, revealing that participants' actual performance did not vary across conditions $(M=10.74)$.

\section{Discussion}

Experiment 2 revealed that perceptions of performance and skill could be influenced by the sequential presentation of feedback. Although all participants received identical feedback overall, variations in the 
sequential presentation of that feedback influenced participants' perceptions of their performance and skill. More important, the specific pattern of results indicated that participants formed on-line self-concepts. That is, their perceptions of performance and skill reflected a heavy emphasis on initial information, which is supportive of on-line self-concept formation.

This pattern of results was anticipated because Experiment 1 revealed that people expect consistency in their own behaviors. These findings, coupled with the literature on on-line impression formation (e.g., McConnell et al., 1994b, 1997b), led us to anticipate the primacy effects observed in the current experiment. Although Experiment 2 is supportive of this reasoning, it would be necessary to manipulate perceptions of consistency for the self to establish its causal role. Hence, Experiment 3 was conducted to experimentally evaluate this hypothesis.

\section{Experiment 3: The causal role of consistency in self- concept formation}

The current experiment manipulated participants' expectations of consistency on analogy items to experimentally assess how perceptions of consistency for the self affect self-concept formation. We expected that those who anticipated consistent performance would reveal evidence of on-line self-concepts, replicating Experiment 2. On the other hand, when participants were induced to expect little consistency in their performance on analogy items, we expected the elimination of these primacy effects.

\section{Method}

\section{Participants}

A sample of 127 undergraduates participated in partial fulfillment of a class requirement. During debriefing, four participants reported suspicion about the noncontingent feedback and were therefore excluded from data analyses. Analyses including these suspicious participants produced identical results.

\section{Procedure and design}

Procedure, materials, and dependent measures were identical to Experiment 2 with two exceptions. First, instructions were presented prior to the beginning of the analogy task to manipulate the perceived consistency of performance on the analogy task. In the consistency condition, participants were told, "In general, most people exhibit very stable, highly consistent performance on analogy items." In the inconsistency condition, participants were told, "In general, most people exhibit very variable, highly inconsistent performance on analogy items." All other instructions were identical to Experiment 2.
Second, two items were included to assess the effectiveness of the consistency manipulation. These manipulation checks were presented at the end of the experiment. The first question assessed participants' memory for the consistency instructions by asking, "How consistent and stable is people's performance supposed to be on analogy items (according to the instructions you read)?" The second item assessed participants' beliefs that people, in general, show consistency on analogy items by asking, "How consistent and stable do you think people's performance is on analogy items?" Each question was answered using on a 9-point scale ranging from 1 (very inconsistent) to 9 (very consistent).

Participants were randomly assigned to conditions in a 2 (consistency instruction: consistent vs. inconsistent) $\times 3$ (presentation condition: primacy, balanced, or recency) factorial design. ${ }^{1}$

\section{Results}

It was expected that when performance on the analogy task was expected to be consistent, participants would form on-line self-concepts, revealed by a strong emphasis on early information. This pattern would replicate Experiment 2. However, when performance on the analogy task was expected to be inconsistent later information should be more influential on judgments. Therefore, the critical prediction is a consistency instruction by presentation condition interaction for participants' performance and skill estimates.

\section{Manipulation check}

To examine whether the manipulation of consistency was effective, 2 (consistency instruction: consistent vs. inconsistent $) \times 3$ (presentation condition: primacy, balanced, or recency) ANOVAs were conducted on the memory for the consistency instructions and on the belief in analogy consistency scores. The predicted main effect of consistency instruction for the memory of the consistency instructions, $F(1,121)=50.13, p<.001$, and for the belief in consistency, $F(1,121)=10.30$, $p<.001$, were both observed. Participants in the consistency condition recalled that the instructions suggested greater consistency $(M=6.49)$ than did those in the inconsistency condition $(M=4.01)$. Likewise, participants in the consistency condition reported a stronger belief in consistency of performance on analogies $(M=5.25)$ than did those in the inconsistency condition $(M=4.44)$. No other effects were observed, $F \mathrm{~s}<1$.

\footnotetext{
${ }^{1}$ After removal of suspicious participants, there were 19 participants in the consistency-primacy condition, 20 participants each in the inconsistency-primacy and inconsistency-recency conditions, 21 participants each in the inconsistency-balanced and consistency-balanced conditions, and 22 participants in the consistency-recency condition.
} 
These findings indicate that the consistency manipulation was effective.

\section{Performance estimate}

A consistency instruction by presentation condition ANOVA was conducted on participants' performance estimates. A marginal main effect of consistency instruction was observed, $F(1,121)=3.32, p<.08$, indicating that those in the consistency condition tended to provide greater performance estimates $(M=16.54)$ than did those in the inconsistency condition $(M=15.73)$. A main effect of presentation condition was also observed, $F(2,121)=7.79, p<.001$, indicating that participants in the primacy condition provided significantly greater performance estimates $(M=17.26)$ than did participants in the balanced $(M=16.05)$ and recency $(M=15.09)$ conditions, which did not differ from each other. More important, these main effects were qualified by the predicted interaction, $F(2,121)=3.42, p<.05$. Means are presented in Table 4.

Simple main effects analyses were conducted to examine whether there was a differential reliance on early versus late information in each of the consistency conditions. There was a main effect of presentation condition in the consistency condition, $F(2,59)=13.70$, $p<.001$, and estimates of analogy performance were significantly different at each level of presentation condition. Specifically, participants in the primacy condition showed the greatest estimate of performance, followed by those in the balanced condition, who in turn showed greater estimates of performance than did those in the recency condition. This strong reliance on early information in the consistency condition indicates that on-line self-concept formation occurred when performance on the analogy task was expected to be consistent. However, there were no differences as a function of presentation condition in the inconsistency condition, $F(2,58)=.46, n s$. When performance was expected to be inconsistent, participants did not utilize relatively early information in their self-judgments.

Table 4

Performance and skill means as a function of consistency expectation condition and presentation condition in Experiment 3

\begin{tabular}{llll}
\hline & \multicolumn{3}{l}{ Presentation condition } \\
\cline { 2 - 4 } Measure & Primacy & Balanced & Recency \\
\hline Perceived performance & & & \\
$\quad$ Expect consistency & $18.31_{\mathrm{a}}$ & $16.57_{\mathrm{b}}$ & $14.73_{\mathrm{c}}$ \\
$\quad$ Expect inconsistency & $16.20_{\mathrm{a}}$ & $15.52_{\mathrm{a}}$ & $15.45_{\mathrm{a}}$ \\
Perceived skill & & & \\
$\quad$ Expect consistency & $6.90_{\mathrm{a}}$ & $6.33_{\mathrm{a}}$ & $5.59_{\mathrm{b}}$ \\
Expect inconsistency & $5.70_{\mathrm{a}}$ & $6.05_{\mathrm{a}}$ & $5.70_{\mathrm{a}}$ \\
\hline
\end{tabular}

Note. Means in a row that do not share a common subscript differ at the .05 level based on Fisher's PLSD tests.
Ability estimate

A consistency instruction by presentation condition ANOVA was conducted on participants' ability estimates. A main effect of consistency instruction was found, $F(1,121)=4.90, p<.05$, showing that participants in the consistency condition reported greater ability at solving analogy items $(M=6.27)$ than did participants in the inconsistency condition $(M=5.28)$. A main effect of presentation condition was also observed, $F(2,121)=3.84, p<.05$, indicating that participants in the primacy $(M=6.30)$ and balanced $(M=6.19)$ conditions reported significantly greater ability estimates than did participants in the recency condition $(M=5.65)$. However, as predicted, these effects were qualified by the two-way interaction, $F(2,121)=3.42, p<.05$.

Table 4 reports a pattern consistent with participants' performance estimates. Simple main effects analyses found an effect of presentation condition in the consistency condition, $F(2,59)=8.68, p<.001$. Specifically, participants in the primacy and balanced conditions reported greater estimates of ability than did those in the recency condition. This strong reliance on early information indicates on-line self-concept formation for those induced to expect consistency in their behaviors. However, as with performance estimates, on-line selfconcept formation was not evident for those in the inconsistency condition, $F(2,58)=.52$, ns. Participants did not emphasize relatively early information in their judgments of analogy skill when they expected inconsistent performance.

\section{Objective performance}

To rule out the possibility that participants' reports of performance and skill were due to objective differences in performance, the number of actual correct responses was analyzed in a consistency instruction by presentation condition ANOVA. Neither main effect approached significance, $F \mathrm{~s}<1$. However, there was an unexpected two-way interaction, $F(2,121)=4.53$, $p<.01$. Follow-up analyses showed that there was a main effect of presentation condition in the consistency condition, $F(2,59)=3.83, p<.05$. Participants in the recency condition performed better on the analogy items $(M=11.09)$ than those in the balanced $(M=8.81)$ condition (neither group differed from those in the primacy condition, $M=10.58$ ). There were no effects of presentation condition in the inconsistency condition, $F(2,58)=1.27, n s$.

The pattern of data in the consistency condition for actual performance (i.e., best actual performance in the recency condition) did not match the pattern observed in participants' reports of performance and skill (i.e., poorest estimates of performance and skill in the recency condition), suggesting that their estimates were not reflective of their actual performance. To rule out this 
possibility inferentially, analyses of covariance (ANCOVAs) were conducted on participants' estimates of performance and skill while controlling for their objective performance. The ANCOVA results were identical to those obtained in the ANOVAs. For performance estimates, the main effect of consistency condition was still marginally significant, $F(1,121)=3.40, p<.07$, and the main effect of presentation condition was still significant, $F(2,121)=8.19, p<.001$. Importantly, their interaction was still significant, $F(2,121)=4.20, p<.05$, even when controlling for objective performance. For ability estimates, the main effect of consistency condition was still significant, $F(1,121)=4.95, p<.05$, as was the main effect of presentation condition, $F(2,121)=4.08$, $p<.05$. More important, their interaction was still significant, $F(2,121)=3.57, p<.05$. Thus, it does not appear that objective performance was responsible for the differences observed in participants' performance and skill estimates.

\section{Discussion}

Experiment 3 provided an empirical test of the reasoning that expectations of consistency for the self influence self-concept formation processes. Indeed, when participants expected consistency in their own behaviors, on-line self-concept formation was observed (replicating Experiment 2). However, when participants were induced to expect inconsistency in their behaviors, on-line self-concept formation was eliminated. Thus, the current experiment tested the proposed explanation for why the results in Experiment 2 obtained. Indeed, expectations about consistency for the self seem to determine whether self-concepts are formed on-line.

One unexpected finding in the current experiment was the interaction observed in participants' objective performance on the analogy items. This pattern of results did not mirror participants' estimates of performance and skill. Also, analyses showed that the predicted interactions between consistency expectancy and presentation condition persisted even when controlling for objective performance. This indicates that the differences in participants' estimates of their own performance and skill were unrelated to their objective performance. Because differences in objective performance were not observed in any other experiments (Experiment 2 and others that we have conducted in our lab), we would conclude that the observed differences in objective performance were probably stochastic in nature. Also, the experimental procedure used in Experiment 4 (to be discussed) was not subject to this concern.

The current work indicates that people expect a great deal of consistency in their behaviors (Experiment 1) and accordingly form on-line self-concepts (Experiment 2) unless they do not anticipate consistency in their behaviors (Experiment 3). Evidence for these conclusions is based on stronger primacy effects being revealed in conditions where on-line self-concept formation was hypothesized. Although relying on primacy effects to infer on-line self-concept formation is sensible, it is also the case that more direct evidence would provide more compelling support for the role of on-line and memorybased judgments. The literatures that examine on-line and memory-based judgments differences have relied on recall-based evidence of social information processing such as amount of recall and memory-judgment correlations (e.g., Hastie \& Park, 1986; McConnell, 2001; McConnell et al., 1994b, 1997b). Experiment 4 collected more direct evidence of the hypothesized underlying processes (i.e., amount of free recall, memory-judgment correlations) as well as examined primacy versus recency effects in self-judgments. Moreover, it also examined the necessity of cognitive resources for on-line self-concepts to occur.

\section{Experiment 4: Cognitive resources and recall-based evi- dence of self-concept processes}

Unfortunately, the analogy task paradigm makes it difficult to assess recall-based measures of judgment processes. Indeed, there are no behavioral data for participants to integrate and to recall except for the feedback they receive after each analogy item. Because of this limitation, we developed a new method that allowed us to experimentally associate self-relevant behaviors to participants. This inkblot paradigm provided participants with a series of inkblots to evaluate. As Fig. 2 illustrates, participants selected from one of five interpretations to characterize the inkblot, and after providing their response, they were provided with a behavior that purportedly describes people who selected that particular description for the inkblot. The behaviors provided were unrelated to participants' choices but instead were part of a between-subjects manipulation designed to influence their self-perceptions of a personality trait (i.e., extraversion). We chose extraversion because pretest participants indicated that they did not have strong beliefs about the extent to which they were outgoing and because previous work found extraversion amenable to experimental manipulation (Fazio, Effrein, $\&$ Falender, 1981). Also, we wanted to extend our work beyond perceptions of performance on analogy items and examine whether these processes would influence self-perceptions about a personality trait.

Similar to the analogy task paradigm, we manipulated the sequential presentation of extraversion-related feedback. Participants in the primacy condition received the majority of their extraverted behaviors early during the task, whereas participants in the recency condition received the majority of their extraverted behaviors later during the task. After completing the inkblot task, 


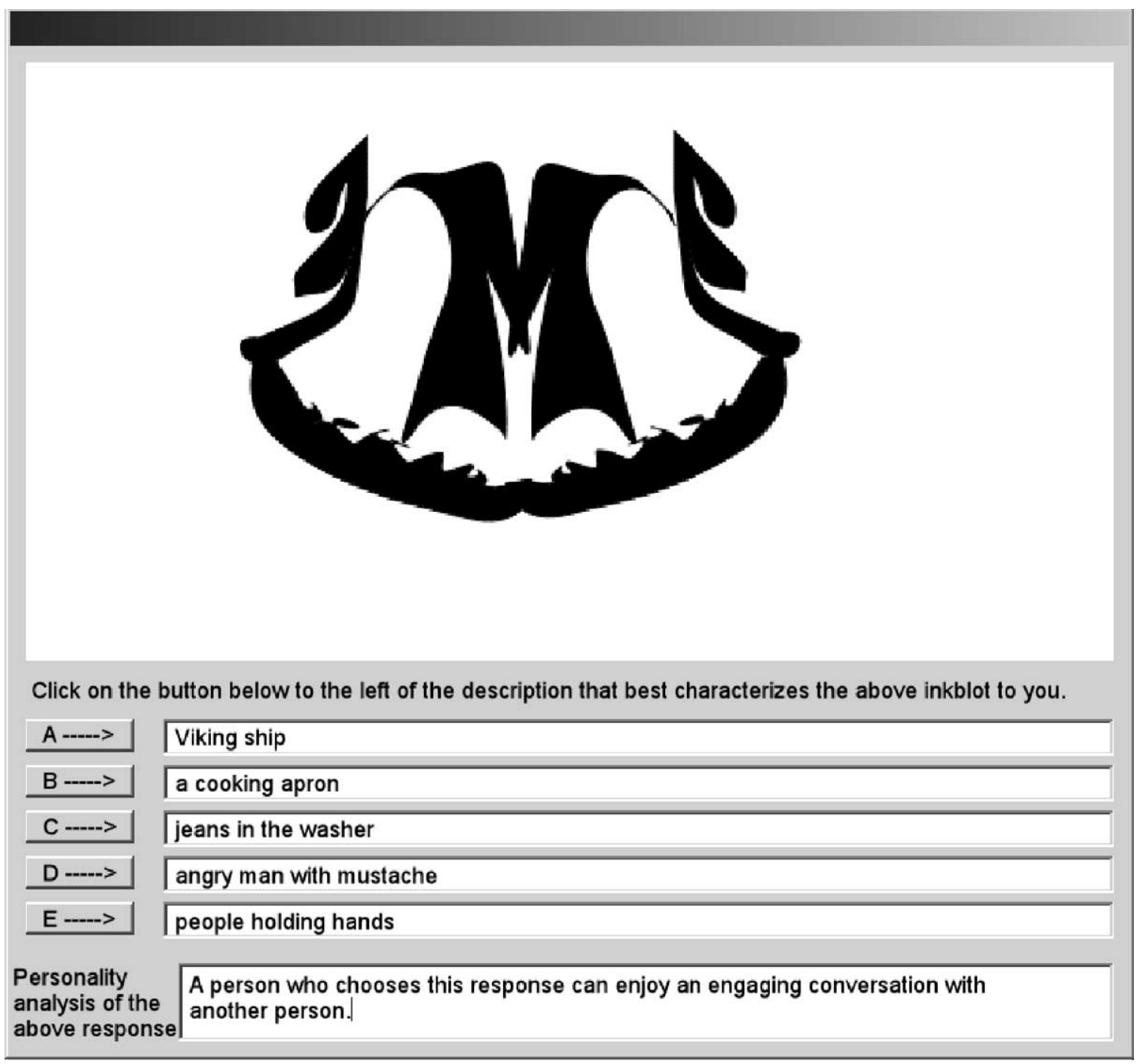

Fig. 2. Interface used for inkblot paradigm task in Experiment 4.

participants were given a surprise free recall test about the self-relevant behaviors that were presented to them. Later, they reported how extraverted they were. If participants form on-line self-concepts, they should report greater estimates of extraversion in the primacy condition than in the recency condition, similar to Experiments 2 and 3. More important, because participants in the inkblot paradigm were provided with self-relevant behaviors, we examined evidence of on-line and memory-based judgments in their free recall. Specifically, we expected that participants would recall more self-relevant behaviors in conditions where on-line self-concepts were expected, reflecting the greater elaboration and information integration that occurs for on-line judgments (McConnell et al., 1994b, 1997b; Srull et al., 1985). Also, we examined whether there were relations between the content of their free recall and their selfjudgments of extraversion. Only memory-based selfconcepts should result in significant memory-judgment correlations (Hastie \& Park, 1986; McConnell, 2001; McConnell et al., 1994b, 1997b).

To explore a range of conditions that are likely to produce on-line and memory-based self-concepts, we manipulated processing resources in Experiment 4. Because forming integrative impressions requires sizable processing resources (Srull, 1981), introducing additional tasks that require short-term memory resources should make it more difficult to concurrently integrate self-relevant information on-line, making memory-based judgments more likely. To manipulate cognitive resources, some participants were asked to keep a twodigit (low load) or a six-digit (high load) number in memory while evaluating each inkblot. A third group (control) was not asked to keep a number in memory. Because keeping longer strings of digits in memory decreases available processing resources (e.g., Bargh \& Tota, 1988) that would be necessary for forming integrative, on-line self-concepts, we anticipated that those 
under high load would reveal memory-based self-concepts (i.e., recency effects in their assessments of extraversion, poorer recall, and memory-judgment correlations). Those under no load should show evidence of on-line self-concepts (i.e., primacy effects in their assessments of extraversion, better recall, and no memory-judgment correlations). Our predictions for the low load group was that they would fall in between the high load and no load conditions, although we did not have a strong a priori belief about whether their more modest resource depletion would induce memory-based self-concepts.

\section{Method}

\section{Participants and design}

A sample of 108 undergraduates participated in partial fulfillment of a class requirement. They were randomly assigned in a 2 (feedback condition: primacy vs. recency) $\times 3$ (cognitive load: no load, low load, or high load) between-subjects factorial design. ${ }^{2}$ None of them reported any suspicion about the study or the feedback provided.

\section{Stimulus materials}

We developed a series of 24 original, black-and-white inkblot figures and asked pretest participants (none of whom took part in the primary study) to suggest descriptions for each inkblot (e.g., an empty flower vase, face of a shaggy dog). The experimenters selected five probable yet distinctive responses for each inkblot, and pretests indicated that undergraduates saw each of them as a viable description for each inkblot. Because the presentation of extraversion-related behaviors was unrelated to the actual response chosen by participants for each inkblot, our primary concern was to provide participants with an engaging activity in which the selection of inkblot interpretations and the presentation of selfrelevant behaviors would be perceived as meaningful to them.

In addition to developing inkblot materials during pretesting, a pool of 16 self-relevant behaviors that suggested extraversion (e.g., "A person who chooses this response can enjoy an engaging conversation with another person.") and 8 self-relevant behaviors that were unrelated to extraversion (e.g., "A person who chooses this responses often prefers complex to simple problems.") were developed for use in the primary experiment.

\footnotetext{
${ }^{2}$ The current experiment did not include a balanced condition because serial position effects (i.e., primacy and recency effects in judgments) are most clearly revealed by comparisons between the two most extreme (i.e., primacy and recency) conditions.
}

\section{Procedure}

Participants arrived at the laboratory, completed consent forms, were randomly assigned to conditions, and completed the remainder of the experiment alone at computer workstations in individual rooms. They were told that the experimenters had developed a new personality test that was based on people's visual perceptions and that the test was accurate and effective in describing people's personalities. They were told that they would evaluate a series of inkblots, and for each, they would indicate which of five responses for each inkblot best described it. Also, they were told that after each response, they would receive feedback about their personality based on their choice. Participants were told that in addition to evaluating inkblots that they should also pay attention to their personality feedback (i.e., the self-relevant behaviors). They were not informed about any judgments that they would later make or that they would be asked to recall the self-relevant behaviors.

Participants studied each inkblot and indicated which one of the five responses they thought best characterized the inkblot. They were allowed to take as much time as they wanted in making their selection. After indicating their choice, a self-relevant behavior (based on condition assignment) was presented on the screen for $8 \mathrm{~s}$ before the next inkblot appeared.

All 24 of the self-relevant behaviors were presented to the participants, but the order of presentation varied based on feedback condition. Similar to the analogy task paradigm, participants in the primacy condition were presented with 10 extraverted and 2 unrelated behaviors during the first block of 12 inkblots and then were presented with 6 extraverted and 6 unrelated behaviors during the last block of 12 inkblots. Participants in the recency condition were presented with 6 extraverted and 6 unrelated behaviors during the first block of 12 inkblots and then were presented with 10 extraverted and 2 unrelated behaviors during the last block of 12 inkblots. The computer randomly selected which extraverted and which unrelated behaviors were presented from their respective lists.

\section{Load manipulation}

Participants in the low load and in the high load conditions were asked to retain a number string in memory while evaluating each inkblot under the pretense of evaluating how engaging in multiple tasks affects personality assessment with the inkblot test. After indicating their response to an inkblot and after reading the self-relevant behavior purportedly associated with their response, participants were asked to enter their digit string on the keyboard. Those in the high load condition were asked to memorize a different six-digit string for each inkblot trial, whereas those in the low load condition were asked to memorize a different twodigit string for each inkblot trial. Participants in the no 
load condition were neither asked to keep anything in memory nor asked to recall anything at the end of each inkblot trial.

\section{Measures}

After completing the 24 inkblots, participants were given a surprise recall task. They were asked to remember as many of the self-relevant behaviors as they could recall, and they were given $8 \mathrm{~min}$ to list them on a sheet of paper. Afterwards, participants were asked to provide ratings ranging from 0 (not at all characteristic of me) to 10 (extremely characteristic of me) on a series of traits (e.g., honest, cynical), including the one of interest, outgoing. ${ }^{3}$ After providing the ratings, participants were fully debriefed and thanked for their participation.

\section{Results}

It was predicted that greater cognitive load would inhibit integrative information processing about the self. Thus, as cognitive load increased, self-concept formation should be less on-line and more memory-based. This shift toward memory-based judgments should lead to greater recency effects in judgments about the self, poorer recall of self-relevant behaviors, and significant memory-judgment correlations.

\section{Trait estimates}

A 2 (presentation condition: primacy vs. recency) $\times 3$ (cognitive load: no load, low load, or high load) ANOVA was conducted on participants' estimates of outgoingness. The only effect to obtain was the predicted interaction between presentation condition and cognitive load, $F(2,102)=7.46, p<.001$, which is presented in Fig. 3. A primacy effect in the no load condition was found, revealed by greater estimates of outgoingness in the primacy condition than in the recency condition, $t(34)=2.14, p<.04$. Further, there was a recency effect in the high load condition, indicated by greater estimates of outgoingness in the recency condition than in the primacy condition, $t(34)=3.24, p<.01$. There was no significant difference between the presentation conditions in the low load condition. Thus, participants showed primacy effects in judgments about themselves under no load conditions, which replicated Experiments 2 and 3 and is consistent with on-line self-concepts. However, under high load, participants showed a recency effect, which is consistent with memory-based selfconcepts when cognitive resources are scant.

\footnotetext{
${ }^{3}$ Pretesting revealed that many undergraduates did not know the definition of "extraversion." When provided with a definition of extraversion, most students said it fit the word "outgoing" best. Thus, we asked participants for estimates of their outgoingness in Experiment 4.
}

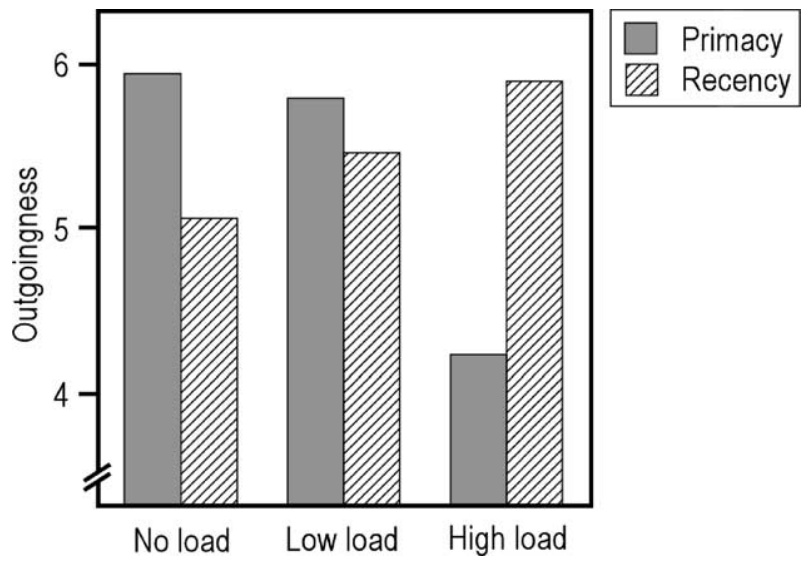

Fig. 3. Interaction between cognitive load and presentation condition for estimates of outgoingness in Experiment 4.

\section{Amount of recall}

Although the effects observed in participants' judgments of their own outgoingness are consistent with the proposed on-line versus memory-based distinction, additional support for this explanation would be revealed if the amount of recall for the self-relevant behaviors was reduced as cognitive load increased. Thus, a main effect of cognitive load was predicted for amount of recall. To examine recall, two trained judges who were unaware of each participant's experimental condition evaluated free recall using gist criterion. They agreed $94 \%$ of the time, and their disagreements were resolved by a third judge (who was also unaware of the experimental conditions).

A presentation condition by cognitive load ANOVA was conducted on the total number of behaviors recalled, and the anticipated main effect of presentation condition was found, $F(2,102)=21.86, \quad p<.001$. Amount of recall did not differ between the no load $(M=14.47)$ and the low load $(M=14.38)$ conditions, but recall in each condition was significantly greater than recall in the high load $(M=11.06)$ condition. No other effects were significant, $F \mathrm{~s}<1$. Thus, when $\operatorname{cog}$ nitive resources were greatly diminished, overall recall was reduced. This finding is consistent with the induction of memory-based judgments (e.g., McConnell, 2001; McConnell et al., 1994b, 1997b).

\section{Memory-judgment correlations}

The recency effects and poorest recall in the high load condition is consistent with memory-based self-concepts. However, the most compelling evidence that memory-based judgments were responsible for the recency effects found in the current experiment would be to observe significant memory-judgment correlations only in the condition where memory-based judgments were apparently induced (i.e., the high load condition). Thus, the number of extraverted behaviors recalled by 
each participant was correlated to that person's ratings of outgoingness for each of the three load conditions. Because memory-based judgments were expected in the high load condition but not in the no load condition, we expected significant memory-judgment correlations in the former but not in the latter. Indeed, significant memory-judgment correlations were observed in the high load condition $(r=.35, p<.04)$, but not in the low load $(r=-.04, n s)$ or in the no load $(r=.14, n s)$ conditions. These results provide strong evidence that memory-based judgments were exhibited only in the high load condition. Indeed, this is the only condition where the strength of participants' ratings of outgoingness was predicted by how many outgoing behaviors they recalled.

\section{Discussion}

Experiment 4 provides strong support that on-line and memory-based judgments are responsible for the observed primacy and recency effects, respectively. When cognitive resources were greatly reduced, recency effects, poor recall, and significant memory-judgment correlations were observed. These findings are hallmark indicators of memory-based judgments (e.g., Hastie \& Park, 1986; McConnell, 2001; McConnell et al., 1997b). On the other hand, when a demanding concurrent task was not present, primacy effects, better recall, and no correspondence between self-judgments and the content of self-relevant recall were found. The differences between the no load and high load conditions provide strong evidence that on-line and memory-based judgments account for the differences observed in our experiments. Experiment 4 also extended our examination of self-relevant information processing into a new behavioral domain using a new experimental task, and comparable results were found. Finding similar outcomes using different methods for judgments of one's ability and of one's personality characteristics adds greater confidence to the generalizability and importance of these phenomena as well as providing greater insight into their underlying causes.

\section{General discussion}

The goal of the current study was to examine some of the basic processes involved in self-concept formation. Although much light has been shed on the implications of the self-concept, far less is known about for how selfrelevant information is processed. We proposed that the on-line versus memory-based distinction could provide insights into understanding the formation and content of self-concepts.

Experiment 1 revealed that people expect consistency in their behavior. In fact, their expectations of consis- tency for the self were either as strong as, or at times even stronger than, their expectations for individuals and always much stronger than for groups. Based on the literature addressing how people form impressions of individuals and groups (Hamilton \& Sherman, 1996; McConnell et al., 1994b, 1997b), we predicted that participants should form on-line self-concepts. Experiment 2 supported this hypothesis by revealing that participants relied on early information in forming impressions about themselves. Experiment 3 provided causal support for the role of perceived consistency for the self. Finally, Experiment 4 collected more direct memory measures using a different paradigm and behavioral domain to provide even stronger support for the hypothesized underlying mechanisms.

The current work not only explored the processes underlying self-concept formation but also demonstrated important consequences for beliefs about the self. As Experiments 2-4 revealed, objectively equivalent information about the self was perceived differently based on how self-concepts were formed. Further, in situations where even the sequential presentation of feedback was identical, such as in the primacy conditions of Experiments 3 and 4, strong differences in selfassessments were observed based on how self-concepts were formed. Thus, the processing mechanisms involved not only affect how self-concepts are formed but also influence self-concept content as well.

In addition to demonstrating the formation of on-line self-concepts, the current work suggests implications for findings in the self literature. For example, Klein et al. (1992) found that individuals retrieved specific instances about themselves for self-relevant qualities that were relatively low in self descriptiveness, but that people retrieved abstracted trait information for aspects of themselves that were highly self descriptive. It is possible that these outcomes may result from the information processing mechanism in self-concept formation. The current work suggests that people are more likely to engage in on-line self-concept formation for domains where consistency for the self is expected.

One of the features of on-line judgments is that an abstracted evaluation is developed early while information is being acquired and actively organized in memory. And as the current study demonstrated, on-line selfconcepts were especially likely for domains where expectations of consistency were strong. If one assumes that highly descriptive self-aspects are also stable selfaspects, one might conclude that the reason why Klein et al. find that people recall traits for highly self-descriptive information is because on-line judgments resulted in the formation of an abstracted trait evaluation produced by integrative processing. And similarly, one would assume that memory-based self-concepts should result in a collection of self-descriptive exemplars. If selfrelevant information that is low in descriptiveness were 
also perceived as relatively inconsistent to an individual, one would expect the recall of specific instances, rather than traits, in these cases. Thus, the recall outcomes that Klein and colleagues obtain may result from whether self-concepts are formed on-line or are memory-based.

The current findings may also speak to why memory for self-relevant information is especially good (e.g., Bower \& Gilligan, 1979). Although the self may not possess unique properties (Greenwald \& Banaji, 1989; Kihlstrom \& Klein, 1994), it is an effective retrieval vehicle because of the greater elaboration of self-relevant information. The current study suggests that selfconcepts quite often will be formed on-line, and that recall is especially good when self-concepts are on-line in nature.

In addition to speaking to findings in the literature regarding the self, the current work also provides useful links to how we form impressions of individuals and develop stereotypes of groups. As noted earlier, work by McConnell et al. (1994b, 1997b) has shown that people typically form on-line judgments about individuals and memory-based judgments about groups (see also, McConnell, Leibold, \& Sherman, 1997a). However, this work has also shown that the type of target encountered is less important than the expectations that one holds about the target and how those expectations induce different processing goals for understanding the target. The current work suggests that the self, as a target of social perception, may have much more in common with impression formation (e.g., warm-cold effects, on-line judgments, especially good recall of impression inconsistent information) than with stereotype development (e.g., illusory correlations, memory-based judgments, and especially good recall of impression consistent information). But more important, by identifying a common process such as the on-line versus memory-based continuum, we can understand how general principles of social perception apply to all social targets. Thus, the current work provides a link between the self literature and programs of research that study impression formation and stereotype development.

Although the current study provides several important insights into how self-relevant information is processed, future work should address other important implications of self-concept formation processes. For instance, because on-line self-concepts will result in people integrating information extensively, on-line selfconcepts should be held with greater confidence and be more resistant to change. Thus, people are likely to cling strongly to important self-relevant beliefs because they are more likely to be formed on-line. This could have especially disturbing consequences for those who suffer from depression or are chronically low in self-esteem.

Relatedly, the current work did not examine how selfconcept formation would occur when self-relevant feedback is negative. The current model would suggest that negative self-concepts might often be formed online, making negative self-regard more likely. However, one of the distinctive properties of the self is that people often are motivated to render self-serving judgments (e.g., Dunning \& Cohen, 1992; Kunda, 1990; Weinstein, 1980). Perhaps people would be less likely to actively integrate and elaborate on negative self-relevant information. On the other hand, negative self-relevant affect may bring more cognitive resources to bear on self-understanding (e.g., Schwarz \& Clore, 1996; Vallacher \& Wegner, 1987), making on-line self-concepts even more likely for self-relevant feedback that induces negative mood. Clearly, these are interesting possibilities for future research to explore.

Yet another area for future research is to study how self-concept formation operates in complex social interaction situations. Because on-line self-concept formation requires cognitive resources, demanding social situations may make resources are scarce. For instance, consider social interactions where one must focus both on impression formation for other people involved in a conversation and on self-concept formation at the same time. These situations will tax available cognitive resources, making on-line judgments more difficult. Although laboratory studies that focus perceivers on only one social target suggest that impression formation and self-concept formation will typically occur on-line, social situations that simultaneously flood perceivers with information relevant to the self and information about others may overwhelm cognitive capacity, inducing memory-based judgments. Even though this suggests that an understanding of social information processing "defaults" may be somewhat oversimplified, the proposed on-line versus memory-based distinction allows us to predict how impressions of the self and about others will be formed under these demanding situations.

In sum, the current work links self-concept formation to an evolving process framework for understanding impression formation for groups and individuals. It found that people have strong expectations of behavioral consistency for the self, and it showed that consistency expectations and processing resources shape self-concept formation and content. The current study may help researchers better understand findings in the literature regarding the amount and content of self-relevant recall, and it may allow psychologists to describe similarities among three core areas in social psychology that have been treated as separate topics (i.e., individuals, groups, and the self). Finally, this distinction suggests several new directions for research that examine the development of self-concepts and how the self is understood. Although it will take additional work to develop a fuller understanding of these phenomena and their implications, the current study takes some initial first steps toward a better understanding of how "know thyself" is accomplished. 


\section{Acknowledgments}

The research was supported by NIMH Grants MH58449 and MH60645. Portions of the work were presented as an invited paper at the 71st Annual Meeting of the Midwestern Psychological Association, Chicago, IL, in April 1999, and at the European Association of Experimental Social Psychology group meeting on Group Entitativity and Group Homogeneity, Université catholique de Louvain, Louvain-laNeuve, Belgium, in July 1999. The authors thank Galen Bodenhausen, John Skowonski, and two anonymous reviewers for their thoughtful suggestions on a previous draft of this article. Also, we appreciate the helpful comments provided by Jim Sherman on an earlier version of this paper and the useful feedback about this work provided by Jeff Sherman. Finally, we thank Corey Bacon, Ellen Carncross, Kristy Dean, Laura Degange, Molly Delaney, Stacy Eppley, Stephanie Epstein, Sarah Evans, John Everhardt, Jessica Fortier, Benjamin Gawle, Sarah Girard, Amy Hughes, Emma Hutter, Amy Johnson, Marcelle Jones, Jessica Misner, Jennifer Shea, and Courtney Snyder for their help in conducting these experiments.

\section{References}

Bargh, J. A., \& Tota, M. E. (1988). Context-dependent automatic processing in depression: Accessibility of negative constructs with regard to the self but not others. Journal of Personality and Social Psychology, 54, 925-939.

Bandura, A. (1986). Social foundations of thought and action: A social cognitive theory. Englewood Cliffs, NJ: Prentice-Hall.

Baxter, T. L., \& Goldberg, L. R. (1987). Perceived behavioral consistency underlying trait attributions to oneself and another: An extension of the actor-observer effect. Personality and Social Psychology Bulletin, 13, 437-447.

Bem, D. J. (1972). Self-perception theory. In L. Berkowitz (Ed.), Advances in experimental social psychology (Vol. 6, pp. 1-62). New York: Academic Press.

Bower, G. H., \& Gilligan, S. G. (1979). Remembering information related to one's self. Journal of Research in Personality, 13, 404419.

Cooley, C. H. (1902). Human nature and the social order. New York: Scribners.

Dreben, E. K., Fiske, S. T., \& Hastie, R. (1979). The independence of evaluative and item information: Impression and recall order effects in behavior-based impression formation. Journal of Personality and Social Psychology, 37, 1758-1768.

Dunning, D., \& Cohen, G. L. (1992). Egocentric definitions of traits and abilities in social judgment. Journal of Personality and Social Psychology, 63, 341-355.

Fazio, R. H., Effrein, E. A., \& Falender, V. J. (1981). Self-perceptions following social interaction. Journal of Personality and Social Psychology, 41, 232-242.

Fiske, A. P., Kitayama, S., Markus, H. R., \& Nisbett, R. E. (1998). The cultural matrix of social psychology. In D. T. Gilbert, S. T. Fiske, \& G. Lindzey (Eds.), Handbook of social psychology (Vol. 2, 4th ed., pp. 915-981). New York: McGraw-Hill.
Greenwald, A. G., \& Banaji, M. R. (1989). The self as a memory system: Powerful, but ordinary. Journal of Personality and Social Psychology, 57, 41-54.

Hamilton, D. L., \& Gifford, R. K. (1976). Illusory correlation in interpersonal perception: A cognitive basis of stereotypic judgments. Journal of Experimental Social Psychology, 12, 392-407.

Hamilton, D. L., \& Sherman, S. J. (1996). Perceiving individuals and groups. Psychological Review, 103, 336-355.

Hastie, R., \& Park, B. (1986). The relationship between memory and judgment depends on whether the judgment task is memory-based or on-line. Psychological Review, 93, 258-268.

Heider, F. (1958). The psychology of interpersonal relations. Hillsdale, NJ: Erlbaum.

Higgins, E. T. (1997). Beyond pleasure and pain. American Psychologist, 52, 1280-1300.

Jones, E. E., \& Nisbett, R. E. (1972). The actor and the observer: Divergent perceptions of the causes of behavior. In E. E. Jones, D. E. Kanouse, H. H. Kelley, R. E. Nisbett, S. Valins, \& B. Weiner (Eds.), Attribution: Perceiving the causes of behavior (pp. 79-94). Morristown, NJ: General Learning Press.

Kihlstrom, J. F., \& Klein, S. B. (1994). The self as a knowledge structure. In R. S. Wyer \& T. K. Srull (Eds.), Handbook of social cognition (Vol. 1, (2nd ed., pp. 153-208). Hillsdale, NJ: Erlbaum.

Klein, S. B., \& Loftus, J. (1990). The role of abstract and exemplarbased knowledge in self-judgments: Implications for a cognitive model of the self. In T. K. Srull \& R. S. Wyer (Eds.), Advances in social cognition (Vol. 3, pp. 131-139). Hillsdale, NJ: Erlbaum.

Klein, S. B., \& Loftus, J. (1993). The mental representation of trait and autobiographical knowledge about the self. In T. K. Srull \& R. S. Wyer (Eds.), Advances in social cognition (Vol. 5, pp. 1-49). Hillsdale, NJ: Erlbaum.

Klein, S. B., Loftus, J., Trafton, J. G., \& Fuhrman, R. W. (1992). Use of exemplars and abstractions in trait judgments: A model of trait knowledge about the self and others. Journal of Personality and Social Psychology, 63, 739-753.

Klein, S. B., Sherman, J. W., \& Loftus, J. (1996). The role of episodic and semantic memory in the development of trait self-knowledge. Social Cognition, 14, 277-291.

Kunda, Z. (1990). The case for motivated reasoning. Psychological Bulletin, 108, 480-498.

Lichtenstein, M., \& Srull, T. K. (1987). Processing objectives as a determinant of the relationship between recall and judgment. Journal of Experimental Social Psychology, 23, 93-118.

Markus, H., \& Kitayama, S. (1991). Culture and the self: Implications for cognition, emotion, and motivation. Psychological Review, 98, 224-253.

Markus, H., \& Kunda, Z. (1986). Stability and malleability of the selfconcept. Journal of Personality and Social Psychology, 51, 858-866.

McConnell, A. R. (2001). Implicit theories: Consequences for social judgments of individuals. Journal of Experimental Social Psychology, 37, 215-227.

McConnell, A. R., Leibold, J. M., \& Sherman, S. J. (1997a). Withintarget illusory correlations and the formation of context-dependent attitudes. Journal of Personality and Social Psychology, 73, 675686.

McConnell, A. R., Sherman, S. J., \& Hamilton, D. L. (1994a). Illusory correlations in the perception of groups: An extension of the distinctiveness-based account. Journal of Personality and Social Psychology, 67, 414-429.

McConnell, A. R., Sherman, S. J., \& Hamilton, D. L. (1994b). On-line and memory-based aspects of individual and group target judgments. Journal of Personality and Social Psychology, 67, 173-185.

McConnell, A. R., Sherman, S. J., \& Hamilton, D. L. (1997b). Target entitativity: Implications for social information processing. Journal of Personality and Social Psychology, 72, 750-762.

Mead, G. H. (1934). Mind, self, and society. Chicago: University of Chicago Press. 
Mischel, W., \& Shoda, Y. (1995). A cognitive-affective system theory of personality: Reconceptualizing situations, dispositions, dynamics, and invariance in personality structure. Psychological Review, 102, 246-268.

Pelham, B. W. (1995). Self-investment and self-esteem: Evidence for a Jamesian model of self-worth. Journal of Personality and Social Psychology, 69, 1141-1150.

Renaud, J. M., \& McConnell, A. R. (2002). Organization of the selfconcept and the suppression of self-relevant thoughts. Journal of Experimental Social Psychology, 38, 79-86.

Rogers, T. B., Kuiper, N. A., \& Kirker, W. S. (1977). Self-reference and the encoding of personal information. Journal of Personality and Social Psychology, 35, 677-688.

Schwarz, N., \& Clore, G. L. (1996). Feelings and phenomenal experiences. In E. T. Higgins \& A. W. Kruglanski (Eds.), Social psychology: Handbook of basic principles (pp. 433-465). New York: Guilford.

Sherman, S. J., Zehner, K. S., Johnson, J., \& Hirt, E. R. (1983). Social explanations: The role of timing, set, and recall on subjective likelihood estimates. Journal of Personality and Social Psychology, $44,1127-1143$.

Shoda, Y., Mischel, W., \& Wright, J. C. (1994). Intra-individual stability in the organization and patterning of behavior: Incorporating psychological situations into the idiographic analysis of personality. Journal of Personality and Social Psychology, 67, 674-687.

Stangor, C., \& McMillian, D. (1992). Memory for expectancycongruent and expectancy-incongruent information: A review of the social and social developmental literatures. Psychological Bulletin, 111, 42-61.

Swann, W. B., Stein-Seroussi, A., \& Giesler, R. B. (1992). Why people self-verify. Journal of Personality and Social Psychology, 62, 392401.

Srull, T. K. (1981). Person memory: Some tests of associative storage and retrieval models. Journal of Experimental Psychology: Human Learning and Memory, 7, 440-463.

Srull, T. K., Lichtenstein, M., \& Rothbart, M. (1985). Associative storage and retrieval processes in person memory. Journal of Experimental Psychology: Learning, Memory and Cognition, 11, 316-345.

Srull, T. K., \& Wyer, R. S. (1989). Person memory and judgment. Psychological Review, 96, 58-83.

Taylor, S. E., \& Brown, J. D. (1988). Illusion and well-being: A social psychological perspective on mental health. Psychological Bulletin, 103, 193-210.

Trope, Y., \& Efrat, N. (1994). Reconciling competing motives in selfevaluation: The role of self-control in feedback seeking. Journal of Personality and Social Psychology, 66, 646-657.

Vallacher, R. R., \& Wegner, D. M. (1987). What do people think they're doing. Action identification and human behavior. Psychological Review, 94, 3-15.

Weinstein, N. D. (1980). Unrealistic optimism about future life events. Journal of Personality and Social Psychology, 39, 806-820.

Wills, T. A. (1981). Downward comparison principles in social psychology. Psychological Bulletin, 90, 245-271. 